\title{
Vereinigte Medizinische Gesellschaft in Kiew Sektion für
}

\section{Augenheilkunde}

\section{Sitzung vom 14. Februar 1928}

Vorsitzender: Priv.-Doz. /. Ginsburg.

1. Powalija: Keratitis proîunda (parenchymatosa circumscripta). Vor zwei Monaten verlor der Kranke sein Sehvermögen auf dem rechten Auge. Auf der Hornhaut des nach einem Trauma erblindeten Auges befindet sich in der Mitte eine Trübung rundlicher Form; im unteren Teil sind noch zwei Trübungen.

In der Diskussion äußerten sich Korenjewitsch, Fridmann, Ginsburg zugunsten von Keratitis disciformis, während Wassütinsky diesen Fall als Keratitis profunda ansehen möchte.

Vereinigte Medizinische Gesellschaft in Kiew. 409

D. Dobrinow. Seltener Fall bösartiger Augengesehwulst. Demonstration eines Kindes mit großer, den ganzen linken Bulbus durchwachsender Geschwulst von Faustgröße. Die Geschwulst erwies sich als Gliom.

D. Gleser: Demonstration eines Kranken nach der Exenteratio orbito-sinualis (wegen Melanosarkom).

A. Wassütinsky: Zur Frage der Extraktion der Katarakt mit Naht. Mitteilung über zwanzig durchgemachte Operationen nach der schon früher beschriebenen Methode mit Naht (vgl. Z. f. A. B. 64, H. 1-2).

Im Laufe des letzten Halbjahres wurden alle Extraktionen in der Augenabteilung des klinischen Oktober schen Krankenhauses immer nach dieser Methode operiert. Dieses Verfahren hat den Vorzug, daß es die Operation fast unabhängig vom Benehmen des Kranken macht (Schauen aufoder abwärts, Unruhigsein, Liderzusammenpressen usw.). Die Naht, sowie die doppelte Fixation soil immer angewandt werden. Obwohl die Extraktionen immer ohne Iridektomie gemacht worden waren, traten niemals Prolapsus iridis oder irgendwie andere Komplikationen auf. Mit den erzielten Erfolgen ist der Vortragende sehr zufrieden.

Diskussion: Korenjewitsch, Salunsky, Ginsburg.

- Sitzung vom 7. März $\lg 28$.

1. M. Charschak demonstriert einen Fall von Exophthalmus infolgeHighmoritis et Ethmoiditis und einen solchen von dauerndem Erf olg nach derWestschen Operation (die Operation wurde vor zwei Jahren ausgeführt).

Aussprache: Schafran, Poljak, Ginsburg.

2. Damskaja: Fall von Luxatio lentis unter der Bindehaut. Nachdem Stoß mit dem Kuhhorn ins linke Auge entstand Luxatio lentis. Dieluxierte Linse befindet sich von außen-oben, nicht weit vom Limbusunter der Konjunktiva. Kolobom der Iris und zyklische Schmerzen.

Aussprache: Korenjewitsch, Ginsburg, Danilewsky, Schafran.

0. Kaganowa: Die Tuberkulose der Iris (von Poljak demonstriert). Das' linke betroffene Auge ist etwas weicher als ein normales; Hornhaut trübe; Präzipitate. In der Vorderkammer höckerige Geschwulst, auf jenem Teil der Iris, der zum Vorschein kommt, Miliartuberkel. WaR. negativ; Pirquet positiv; die Internisten haben Broncho-adenitis fest-gestellt. 
G. Poljak: Doppelseitige angeborene Katarakt-Embryonalnähte der Linse. Pat. 22 Jahre. Auf beiden Augen ist, besonders gut sichtbarbei Be-trachtung mit der Spaltlampe Katarakt der vorderen und hinteren Embryo-nalnähte zu sehen. Nach der Nase zu unterscheidet man Reste von Arteria hyaloidea persistens. Peripherisch findet man in tieferen Schichten der Linse eine Menge punktförmiger Trübungen von hübscher bläulicher Farbe. Diese Erscheinung ist besonders gut auf dem linken Auge ausgeprägt.

5. Grineweísky: Fall von Keratokonus. Spaltlampenbefund: LinkeaAuge: zentrale Hornhauttrübungen, mehr oberflächliche näher zur Peripherie und tieferen im Zentrum. Das Zentrum ist dünner und wölbt sichetwas vor. Im unteren Teil der Hornhaut sind bräunliche Präzipitatezu sehen. Um die Trübungen herum findet man nicht vollständig ge-schlossenen Fleischer schen Ring. Endothelien ohne Veränderungen.

410 Vereinigte Medizinische Gesellschaft in Kiew.

Auf dem rechten Auge dieselben Veränderungen, aber der Ring vollständig geschlossen, und Endothelium mit verwaschenen Grenzen. Mit dem Keratoskop von Placião - unregelmäßige Ringe. Vis. oc. utr. $=o, i$, Infantilismus. Das Interessante dieses Falles besteht darin, daß der Kerato-konus nur bei der Spaltlampenuntersuchung festgestellt werden konnte.

Aussprache: Korenjewitsch, Ginsberg.

6. Dombrowskaja: Tarsitis luetica. Regelmäßige Verdickung des unteren Lidknorpels des rechten Auges, nicht zuheilende Exkoriationen in den Augenwinkeln und purulenter Ausschlag am Halse. WaR. +++. Röntgenoskopisch Periostverdickung der Tibia.

Aussprache : Zalunsky, Zetkowsky, Schafran, Poljak.

$\eta$. J. Salunsky demonstriert: a) Retinitis circinata. b) Corpus alienum in der Linse mit traumatisclier Katarakt. Metallarbeiter bekam einen Hammerschlag ins Auge. Beim Untersuchten traumatische Katarakt; auf der Oberfläche der getrübten Linse ist ein punktförmiges glän-zendes Corpus alienum zu sehen. Auf der Hornhaut kleine lineare Trübung. 8. /. Schewtschuk: Demonstration mikroskopisclier Präparate, die nach Kochal gefärbt sind. Imprägnation mit Gold und Siber.

Poljak.

Diagnose und Therapie.

R. Scheerer (Oberarzt der Univ.-Augenklinik Tubingen), Über einige Er-krankungen des Sehnervenstammes. Arch. f. Augenheilkunde Bd. 99., H. 3, 1928.

S. Seit 1921 wurden in der Klinik (Vorstancl: Prof. W. Stock) etwa 200 Fälle der folgenden Art beobachtet: Bei Patienten von 12 bis 35 Tahren trat plötzlich

vorwiegend einseitig

eine Verdunkelung des Gesichtsfelde $3 / 4$ auf

die von der Mitte aus über das ganze Gesichtsfeld sich verbreitete

nach einigen Tagen in umgekehrter Richtung sich zurückbildete und in drei bis vier Wochen unter Ausbildung einer schläfenseitigen Abblassung der bis dahin nicht nachweisbar veränderten Sehnervenscheibe vollkommcn abheilte. Häufig blieb nur ein kleiner zentraler oder parazentraler Aus-fall für Farben (Rot und Grün) zurück. Das Sehvermògen pflegte sich ganz oder nahezu vollkommen wiederherzustellen. Abweichungen kamen meist nur bei bzgl. der Behandlung verschleppten Fallen vor. Doch war man bei Beginn nicht in der Lage eine sichere Beziehung zwischen Ver-lauf und Behandlung anzunehmen. - Ursachen: In mindestens 55\% der Fälle war multiple Sklerose nachweislich Ursache der Erkrankung. Oft ist die Erkrankung des Sehnerven das erste Zeichen des Leidens 
der erst viel später schwere Erscheinungen an anderen Nerven des Körpers folgen. Bei genauer Nachforschung sind in der Anamnese aber oft Angaben über flüchtige Empfindungs- und Bewegungsstörungen zu erhalten. Keinesfalls bedarf es mehr des Vollbildes der Krankheit nach Charcoi zur Sicher-stellung des Nervenleidens. Wichtig ist die Steigerung aller Beschwerden auch der am Auge

bei körperlicher Anstrengung. (Naheres bei v. Hipp $\beta 1$

Krankheiten des Sehnerven

im Handbuch Graefe-Saemisch.) 